\title{
Digital Option Pricing Approach Using A Homotopy Perturbation Method
}

\author{
Amirul Hakam, Islachiyatul Ummah, Frida Akbar Rani, Nur Asiyah and Endah RM. Putri
}

\begin{abstract}
An option is a financial contract between buyers and sellers. The Black-Scholes equation is the most popular mathematical equation used to analyze the option pricing. The exact solution of the Black-Scholes equation can be approached by several approximation methods, one of the method is a Homotopy Perturbation Method (HPM). The simplest type of option, digital options were analyzed using the HPM. The digital option pricing approach using the HPM is in a power series form, which in this paper is presented the solution in the fourth power. This solution is compared with the exact solution of the Black-Scholes equation for digital options. The results show that the approach using HPM is very accurate.
\end{abstract}

Index Terms-Digital Option, Black-Scholes Equation, Homotopy Perturbation Method.

\section{INTRODUCTION}

O NE of the financial derivative products is options. An option is a financial contract between buyers and sellers. Generally, there are two options types: a call option (a right to buy) and a put option (a right to sell). In the put/call option, holders can buy/sell the underlying asset before the expiry date at a strike price. Considering the exercise date of options, European and American options are usually concerned. European options can be exercised only at the expiry date, whereas American options can be exercised at any time until the expiry date. The most popular mathematical equation used to analyze the option pricing, the Black-Scholes equation, was proposed by Black and Scholes in 1973 [1]. The Black Scholes equation is derived with the following assumptions: the asset price follows a geometric Brownian motion, no dividends, no transaction costs, no taxes and a risk-free interest.

An exact solution of the Black Scholes equation has been obtained [1]. There are various methods to obtain an exact solution approach: Finite Difference Method [2], [3], Monte Carlo Method [4], Quintic B-spline collocation approach [5], Adomian Decomposition Method (ADM) [6], [7], Homotopy Perturbation Method (HPM) [8], [9], [10], etc.

In the European put option, if the strike price $(K)$ exceeds the final asset price $(S(T))$, the put option holder can purchase the asset for $(S(T))$ and immediately sell it to the put writer for $(K)$. The payoff is $S(T)-K$, accordingly. On the contrary, if $S(T)>K$, the put option holder is better off selling the asset at the market price and simply walk away from the contract, and the payoff becomes 0 . Under these conditions,

The authors are with the Department of Mathematics, Faculty of Science and Data Analytics, Institut Teknologi Sepuluh Nopember, Surabaya, Indonesia e-mail: amirul@its.ac.id.

Manuscript received July 19, 2021; accepted July 26, 2021. the payoff from the European put option at final time $(T)$ can be represented as

$$
P(S, T)= \begin{cases}S-K, & S \leq K \\ 0, & S>K\end{cases}
$$

which is a non-differentiable and non-smooth condition at $S=$ $K$. Various approaches are used to solve non-differentiable and non-smooth problems, but a singularity point appears in the derivative. In [7], Ziwie Ke et al. proposed a proper way to apply the ADM to the Black Scholes model for European options. It can solve the singularity problem without requiring a differentiable approximation of the payoff function. By a variable transformation, the singularity is shifted to infinity. Simulation results show that ADM with the variable transformation can solve the singularity problem in the payoff function and provide accurate results.

This paper discusses the Black Scholes solution approach using HPM, where HPM is one of the approach solution methods in a power series, similar to the ADM. Digital options are the simplest type of options. The digital options have a payoff function as European options, which can not be derived at $S=K$. Using the variable transformation introduced in [7], we will apply it with HPM. The outline of this paper is organized as follows. In Section 1, we present the background of the topic. In Section 2 provides a literature review about the basic idea of HPM and a mathematical model for the Digital option. In Section 3 discusses a process to solve the Black Scholes model for the digital options using variable transform and HPM. Then, we simulate the digital option prices and analyze the results. Finally, the conclusion is presented in Section 4.

\section{Research Methods}

\section{A. Homotopy Perturbation Method}

The Homotopy Perturbation Method is one of the potential methods for solving a partial differential equation. The solution obtained from HPM converges rapidly to the exact solution [11], [12]. To understand the basic concept of HPM, consider the following general form

$$
\mathcal{A}(u)-f(r)=0, r \in \Omega,
$$

with boundary condition

$$
\mathcal{B}\left(u, \frac{\partial u(r)}{\partial n}\right)=0, r \in \Gamma .
$$

where $\mathcal{A}$ is a differential operator, $\mathcal{B}$ is a boundary operator, $f(r)$ is a known function, $\Gamma$ is the boundary of a domain $\Omega$ 
and $u(r)$ is a function to be determined and depends on $r$. The operator $\mathcal{A}$ can be divided into two parts $\mathcal{L}$ and $\mathcal{N}$ where $\mathcal{L}$ is a linear operator and $\mathcal{N}$ is a non-linear operator. Therefore, Eq. (1) can be written as follows:

$$
\mathcal{L}(u)+\mathcal{N}(u)-f(r)=0
$$

We construct a homotopy $v(r, p): \Omega \times[0,1] \rightarrow \mathcal{R}$ which satisfies:

$$
\begin{aligned}
& H(v, p)= \\
& \mathcal{L}(v)-\mathcal{L}\left(u_{0}\right)+p[\mathcal{L}(v)+\mathcal{N}(v)-f(\tau)]=0,
\end{aligned}
$$

with $p \in[0,1] . p$ is a embedding parameter and $u_{0}$ is an initial guess of Eq. (1) which satisfies the boundary conditions. By applying a perturbation technique, the solution of Eq. (4) can be assumed in power series form

$$
v=v_{0}+v_{1} p+v_{2} p^{2}+\cdots .
$$

For $p=1$, Eq. (5) can be rewritten as

$$
u=\lim _{p \rightarrow 1} v=v_{0}+v_{1}+v_{2}+\cdots
$$

which is the approximate solution to Eq. (1).

\section{B. Digital Option}

A digital option is a type of options contract that has a fixed payout if the underlying asset moves past the strike price. Option holders buy/sell shares by observing the movement of share prices and strike price. If the share price at maturity on the market is above or higher than the strike price, the holder bought the call option. Instead, if the share price at maturity on the market is below or lower than the strike price, the holder bought the put option.

The digital call option price can be obtained by solving the Black-Scholes equation with a payoff function

$$
\begin{aligned}
C_{t} & =-\frac{1}{2} \sigma^{2} S^{2} C_{S S}-r S C_{S}+r C, \\
C(S, T) & =\left\{\begin{array}{ll}
1 & S>K \\
0 & S \leq K
\end{array} .\right.
\end{aligned}
$$

Corresponding with Eq. (7), The digital put option price satisfies the following mathematical model

$$
\begin{aligned}
P_{t} & =-\frac{1}{2} \sigma^{2} S^{2} P_{S S}-r S P_{S}+r P, \\
P(S, T) & =\left\{\begin{array}{ll}
0 & S>K \\
1 & S \leq K
\end{array} .\right.
\end{aligned}
$$

Both Eq. (7) and (9) with their respective initial conditions can be resolved by similarly as European option exact solution procedures as follows [13]:

1) Make a dimensionless equation with the following variable transformations:

$$
S=K e^{x} ; \quad t=T-\frac{2 \tau}{\sigma^{2}} ;
$$

$C(S, t)=v(x, \tau)$ for Digital call option;

$$
P(S, t)=v(x, \tau) \text { for Digital put option }
$$

2) Make a diffusion equation with the following transformation:

$$
v(x, \tau)=e^{\alpha x+\beta \tau} u(x, \tau)
$$

3) Solve the diffusion equation by Green's function

4) The solution of the third step is exact solution of the Digital call/put option

The digital option exact solution based on the procedures are as follows

$$
\begin{aligned}
& C(S, t)=e^{-r(T-t)} N(d) \\
& P(S, t)=e^{-r(T-t)} N(-d)
\end{aligned}
$$

where

$$
d=\frac{\ln \left(\frac{S}{K}\right)+\frac{1}{2}\left(2 r-\sigma^{2}\right)(T-t)}{\sigma \sqrt{(T-t)}}
$$

and $N($.$) is a cumulative distribution function of the normal$ standard distribution.

The relationship between call options and put options at the same strike price and maturity is called put call parity. The put call parity formula for digital options is given by

$$
C(S, 0)+P(S, 0)=e^{-r T} .
$$

\section{Results And Discussions}

In this section, the mathematical model for the digital option in Eq. (7-10) will be solved by the Homotopy Perturbation Method (HPM). Considering the digital call option, to simplify and non-dimensionalize the model, Eq. (7) is transformed by Eq. (11)

$$
v_{x x}-v_{\tau}+(k-1) v_{x}-k v=0
$$

and the payoff function

$$
v(x, 0)=\left\{\begin{array}{ll}
1 & x>0 \\
0 & x \leq 0
\end{array},\right.
$$

where $k=2 r / \sigma^{2}$. The payoff function in Eq. (8) becomes an initial condition in Eq. (17). When $x=0$, Eq. (17) has a singularity and it is non-differentiable. In order to shift the singularity point to infinity, the transformation is given below [7]:

$$
x=u \sqrt{\tau} ; \quad z=\sqrt{\tau} ; \quad v(x, \tau)=w(u, z) .
$$

Understandably, at $\tau=0$ it changed the $u$ to infinity, as $\lim _{\tau \rightarrow 0} u= \pm \infty$. As a result, Eq. (16) transform to

$$
(w z)_{z}=2 w_{u u}+u w_{u}+w+2(k-1) z w_{u}-2 k w z^{2} .
$$

Also, the initial condition (Eq. (17)) becomes

$$
\lim _{\tau \rightarrow 0} w(u, z)=\left\{\begin{array}{ll}
1 & u \rightarrow+\infty \\
0 & u \rightarrow-\infty
\end{array} .\right.
$$

Now, applied HPM technique to solve the Eq. (19). The parameter $p$ is embedded in Eq. (19)

$$
(w z)_{z}=2 w_{u u}+u w_{u}+w+2(k-1) z p w_{u}-2 k w p^{2} z^{2} .
$$


where $p \in[0,1]$ and Eq. (21) has a solution in the power series as follows

$$
w=w_{0}+p w_{1}+p^{2} w_{2}+\cdots
$$

Consider Eq. (22), we can rewrite Eq. (21) and arrange them according to order of $p$

$$
\begin{aligned}
p^{0}:\left(z w_{0}\right)_{z} & =2\left(w_{0}\right)_{u u}+u\left(w_{0}\right)_{u}+w_{0} \\
p^{1}:\left(z w_{1}\right)_{z} & =2\left(w_{1}\right)_{u u}+u\left(w_{1}\right)_{u}+2(k-1) z w_{0}+w_{1} \\
p^{2}:\left(z w_{2}\right)_{z} & =2\left(w_{2}\right)_{u u}+u\left(w_{2}\right)_{u}+2(k-1) z w_{1}+w_{2} \\
& +2 k z^{2} w_{0} \\
\vdots & \\
p^{n}:\left(z w_{n}\right)_{z} & =2\left(w_{n}\right)_{u u}+u\left(w_{n}\right)_{u}+2(k-1) z w_{n-1} \\
& +w_{n}+2 k z^{2} w_{n-2}
\end{aligned}
$$

To solve Eq. (23) for each $w_{i}$ and satisfy the initial condition in Eq. (20), assumed that $w_{i}(u, z)=f_{n}(u) z^{n}$. Eq. (23) can be written as

$$
\begin{aligned}
& w_{0}=2\left(f_{0}\right)_{u u}+u\left(f_{0}\right)_{u}+f_{0} \\
& w_{1}=\left(2\left(f_{1}\right)_{u u}+u\left(f_{1}\right)_{u}+2(k-1) f_{0}+f_{1}\right) z \\
& w_{2}=\left(2\left(f_{2}\right)_{u u}+u\left(f_{2}\right)_{u}+2(k-1) f_{1}+2 k f_{0}+f_{2}\right) z^{2}
\end{aligned}
$$

$w_{n}=\left(2\left(f_{n}\right)_{u u}+u\left(f_{n}\right)_{u}+2(k-1) f_{n-1}+f_{n}+2 k f_{n-2}\right) z^{n}$

and the initial condition becomes:

$$
\begin{aligned}
& \lim _{\tau \rightarrow 0} w(u, z)=\left\{\begin{array}{ll}
1 & u \rightarrow+\infty \\
0 & u \rightarrow-\infty
\end{array}, \text { for } n=0,\right. \\
& \lim _{\tau \rightarrow 0} w(u, z)=\left\{\begin{array}{ll}
0 & u \rightarrow+\infty \\
0 & u \rightarrow-\infty
\end{array}, \text { for } n \geq 1 .\right.
\end{aligned}
$$

Eq. (24) is a second-order linear ordinary differential equation. For the first term $w_{0}$, let $f_{0}=u s(u)$ to get a general solution as follows:

$$
f_{0}=c_{2}+c_{1} \sqrt{\pi} \operatorname{Erf}\left(\frac{u}{2}\right) .
$$

where $c_{1}$ and $c_{2}$ are two arbitrary constants and $\operatorname{Erf}($.$) is a$ error function. Eq. (27) should satisfy Eq. (25). As a result, a special solution of $f_{0}$ is as follows

$$
f_{0}=\frac{1}{2}\left(1+\operatorname{Erf}\left(\frac{u}{2}\right)\right) .
$$

For $n \geq 1$, each of term $f_{i}$ was solved by using the same procedures as $f_{0}$ and satisfy the Eq. (26).

$$
\begin{aligned}
& w_{0}=\frac{1}{2}\left(1+\operatorname{Erf}\left(\frac{u}{2}\right)\right) \\
& w_{1}=\left[\frac{(k-1)}{2 \sqrt{\pi}} e^{\left.-\frac{u^{2}}{4}\right]}\right] \\
& w_{2}=\left[\frac{1}{4}\left(-\frac{(k-1)^{2} u}{2 \sqrt{\pi}} e^{-\frac{u^{2}}{4}}-2 k\left(1+\operatorname{Erf}\left(\frac{u}{2}\right)\right)\right)\right] z^{2}
\end{aligned}
$$

$$
\begin{aligned}
w_{3} & =\left[\frac { 1 } { 2 4 } \frac { ( k - 1 ) e ^ { - \frac { u ^ { 2 } } { 4 } } } { 2 \sqrt { \pi } } \left(-2+u^{2}+k^{2}\left(u^{2}-2\right)\right.\right. \\
& \left.\left.-2 k\left(u^{2}+10\right)\right)\right] z^{3} \\
w_{4} & =\left[\frac { 1 } { 1 9 2 } \left(e ^ { - \frac { u ^ { 2 } } { 4 } } \left(\frac{6(k-1)^{2}(1+k(k+6) u)}{2 \sqrt{\pi}}\right.\right.\right. \\
& \left.\left.\left.-\frac{(k-1)^{4} u^{3}}{2 \sqrt{\pi}}\right)+48 k^{2}\left(1+\operatorname{Erf}\left(\frac{u}{2}\right)\right)\right)\right] z^{4}
\end{aligned}
$$

Recalled the Black Scholes model for the digital put option price in Eq. (9) with the payoff function (Eq. (10)). To complete the model, the same procedure is applied since the difference only in the payoff function. Therefore, Eq. (9) becomes Eq. (23) and the payoff function becomes an initial condition as follows

$$
\begin{aligned}
& \lim _{\tau \rightarrow 0} w(u, z)=\left\{\begin{array}{ll}
1 & u \rightarrow-\infty \\
0 & u \rightarrow+\infty
\end{array}, \text { for } n=0,\right. \\
& \lim _{\tau \rightarrow 0} w(u, z)=\left\{\begin{array}{ll}
0 & u \rightarrow-\infty \\
0 & u \rightarrow+\infty
\end{array}, \text { for } n \geq 1 .\right.
\end{aligned}
$$

The solution of Eq. (23) satisfying the initial conditions (Eq. $(29-30))$, is as follows

$$
\begin{aligned}
w_{0}= & \frac{1}{2}\left(1-\operatorname{Erf}\left(\frac{u}{2}\right)\right) \\
w_{1}= & {\left[-\frac{(k-1)}{2 \sqrt{\pi}} e^{-\frac{u^{2}}{4}}\right] z } \\
w_{2}= & {\left[\frac{1}{4}\left(-\frac{(k-1)^{2} u}{2 \sqrt{\pi}} e^{-\frac{u^{2}}{4}}-2 k\left(1-\operatorname{Erf}\left(\frac{u}{2}\right)\right)\right)\right] z^{2} } \\
w_{3}= & {\left[-\frac{1}{24} \frac{(k-1) e^{-\frac{u^{2}}{4}}}{2 \sqrt{\pi}}\left(-2+u^{2}+k^{2}\left(u^{2}-2\right)\right.\right.} \\
\left.\left.-2 k\left(u^{2}+10\right)\right)\right] z^{3} & \\
w_{4}= & {\left[\frac { 1 } { 1 9 2 } \left(e ^ { - \frac { u ^ { 2 } } { 4 } } \left(\frac{-6(k-1)^{2}(1+k(k+6) u)}{2 \sqrt{\pi}}\right.\right.\right.} \\
& \left.\left.\left.+\frac{(k-1)^{4} u^{3}}{2 \sqrt{\pi}}\right)+48 k^{2}\left(1-\operatorname{Erf}\left(\frac{u}{2}\right)\right)\right)\right] z^{4} .
\end{aligned}
$$

The solution of put and call option prices using the HPM satisfies the put call parity formula in Eq. (11). This implies that put option price can be obtained by call option price, and vice versa.

The digital option price can be obtained by restoring the variables to their original form. In this paper, we present the Black-Scholes solution approach to the $u_{4}$ form of the power series (Eq. (22)) considering that following the same 


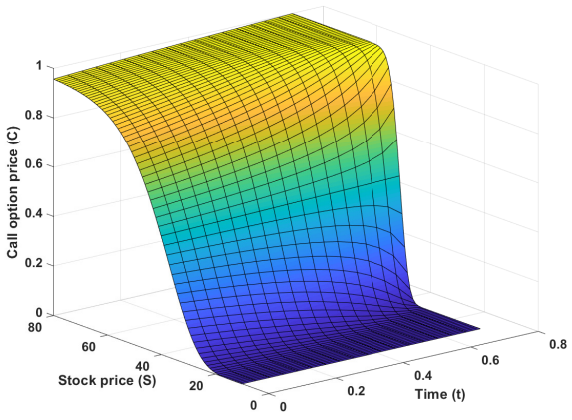

Fig. 1. The Digital call option price solved by HPM.

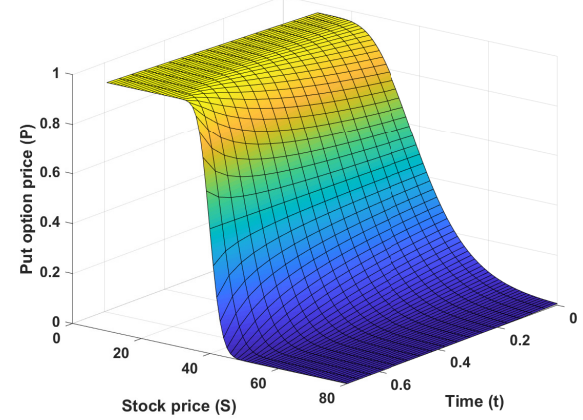

Fig. 2. The Digital put option price solved by HPM.

procedure will lead to the subsequent solution terms. The solution approach using Homotopy Perturbation Method will be simulated and compared with the exact solution of the BlackScholes digital option model. In performing simulation, some parameters have been defined. The strike price $(K)=40$, the interest rate $(r)=0.05$, the volatility $(\sigma)=0.324336$.

The simulation of call and put options price is shown in Figure 1 and Figure 2, respectively. The stock price $(S)$ is given in the interval $[10,80]$ for $T=3 / 4$. The call option price increases as the stock price increases. Instead, the put option price decreases as the stock price increases. When the asset price is greater than the strike price, the digital put option price at maturity date is 0 but the digital call option price towards 1 . It corresponds to the payoff function. At some point in stock prices before expiration, the digital call option price decreases from the price at expiration time and smoothly shifting to the left for $S \leq K$. Similar simulation results also exist for the digital put option. Therefore, based on the simulation, the non-smooth condition of the payoff function of the put/call at $S(T)=K$ can be solved.

The accuracy of HPM for solving Black Scholes models is checked by comparing the results with exact solutions. It is simulated by determining the stock price $S=30,40$, and 50 on the maturity date $T=3 / 4$ and $6 / 12$.

Table I shows the simulation results using HPM and exact solutions for the Digital call option. By looking at Table I, we can see that the results of HPM are very close to the exact solution. The difference is not more than $10^{-5}$ and is shown in
TABLE I

COMPARISON HPM AND EXACT SOLUTION DIGITAL CALL OPTION

\begin{tabular}{|c||c||c||c|}
\hline$S$ & $T$ & Homotopy Perturbation Method & Exact solution \\
\hline \multirow{2}{*}{30} & $3 / 4$ & 0.145673788050671 & 0.145671324096918 \\
\cline { 2 - 4 } & $6 / 12$ & 0.101262455162682 & 0.101261866297924 \\
\hline \multirow{2}{*}{40} & $3 / 4$ & 0.478938990813540 & 0.478932715368402 \\
\cline { 2 - 4 } & $6 / 12$ & 0.485454029537582 & 0.485452035255446 \\
\hline \multirow{2}{*}{50} & $3 / 4$ & 0.755639791967107 & 0.755631538232393 \\
\cline { 2 - 4 } & $6 / 12$ & 0.812735833721423 & 0.812733236242847 \\
\hline
\end{tabular}

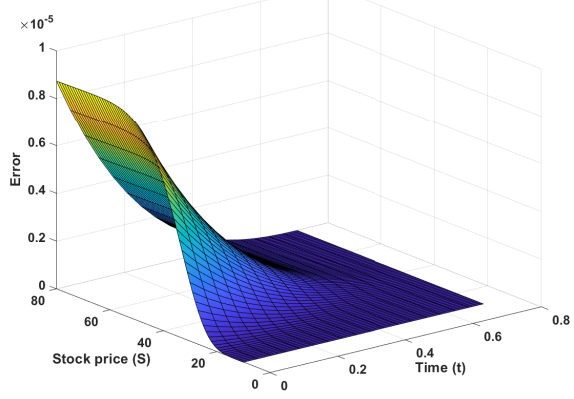

Fig. 3. Difference between HPM and exact solutions of Digital call option.

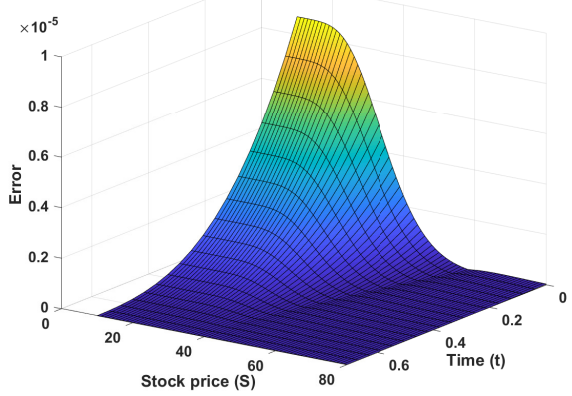

Fig. 4. Difference between HPM and exact solutions of Digital put option.

Figure 3 to display in more detail each point of stock price $(S)$ against time $(t)$, where the most significant difference occurs when $C(80,0)$.

TABLE II

COMPARISON HPM AND EXACT SOLUTION DigITAL PUT OPTION

\begin{tabular}{|c||c||c||c|}
\hline$S$ & $T$ & Homotopy Perturbation Method & Exact solution \\
\hline \multirow{2}{*}{30} & $3 / 4$ & 0.817529336949329 & 0.817523093623904 \\
\cline { 2 - 4 } & $6 / 12$ & 0.874050044837319 & 0.874048045730409 \\
\hline \multirow{2}{*}{40} & $3 / 4$ & 0.484264134186460 & 0.484261702352420 \\
\cline { 2 - 4 } & $6 / 12$ & 0.489858470462418 & 0.489857876772887 \\
\hline \multirow{2}{*}{50} & $3 / 4$ & 0.207563333032893 & 0.207562879488429 \\
\cline { 2 - 4 } & $6 / 12$ & 0.162576666278576 & 0.162576675785486 \\
\hline
\end{tabular}

Comparison of digital put option prices using HPM with exact solutions is shown in Table II. It shows very good results i.e. the results using HPM are close to the exact solution. 
Detail the difference between HPM and the exact solution is shown in Figure 4. Based on this comparison, it can be seen that the difference obtained is also very small, which is not more than $10^{-5}$.

\section{Conclusions}

In this paper, we propose the approximate solution of the Black Scholes model for digital options using HPM. The option has a singularity point at $S=K$. The variable transformation was applied to shift the singularity point to infinity and applied the HPM to solve the Black-Scholes equation after being transformed. The HPM solutions were simulated and compared with the exact solutions. The simulation results show that the variable transformation can solve the singularity problem well and gives very accurate results.

\section{REFERENCES}

[1] F. Black and M. Scholes, "The pricing of options and corporate liabilities," in World Scientific Reference on Contingent Claims Analysis in Corporate Finance: Volume 1: Foundations of CCA and Equity Valuation, 2019, pp. 3-21.

[2] L. Mardianto, A. Pratama, A. Soemarsono, A. Hakam, and E. Putri, "Comparison of numerical methods on pricing of european put options," IJCSAM (International Journal of Computing Science and Applied Mathematics), vol. 5, no. 1, pp. 30-34, 2019.

[3] Z. Cen and A. Le, "A robust and accurate finite difference method for a generalized black-scholes equation," Journal of Computational and Applied Mathematics, vol. 235, no. 13, pp. 3728-3733, 2011.

[4] P. Boyle, "Options: A monte carlo approach," Journal of financial economics, vol. 4, no. 3, pp. 323-338, 1977.

[5] R. Mohammadi, "Quintic b-spline collocation approach for solving generalized black-scholes equation governing option pricing," Computers \& Mathematics with Applications, vol. 69, no. 8, pp. 777-797, 2015.

[6] S. Rodriguez and F. Marin, "European call option pricing by the adomian decomposition method," Advances in Dynamical Systems and Applications, vol. 9, no. 1, pp. 75-85, 2011.

[7] Z. Ke, J. Goard, and S.-P. Zhu, "An appropriate approach to pricing european-style options with the adomian decomposition method," The ANZIAM Journal, vol. 59, no. 3, pp. 349-369, 2018.

[8] E. Putri, L. Mardianto, A. Hakam, C. Imron, and H. Susanto, "Removing non-smoothness in solving black-scholes equation using a perturbation method," Physics Letters A, vol. 402, p. 127367, 2021.

[9] J.-H. He, "Homotopy perturbation method: a new nonlinear analytical technique," Applied Mathematics and computation, vol. 135, no. 1, pp. 73-79, 2003.

[10] V. Gülkaç, "The homotopy perturbation method for the black-scholes equation," Journal of Statistical Computation and Simulation, vol. 80, no. 12, pp. 1349-1354, 2010.

[11] Z. Ayati and J. Biazar, "On the convergence of homotopy perturbation method," Journal of the Egyptian Mathematical society, vol. 23, no. 2, pp. 424-428, 2015.

[12] J. Biazar and H. Aminikhah, "Study of convergence of homotopy perturbation method for systems of partial differential equations," Computers \& Mathematics with Applications, vol. 58, no. 11-12, pp. 2221-2230, 2009.

[13] P. Wilmott, S. Howison, and J. Dewynne, The mathematics of financial derivatives: a student introduction. Cambridge university press, 1995. 